\title{
Budding yeast Hed1 down-regulates the mitotic recombination machinery when meiotic recombination is impaired
}

\author{
Hideo Tsubouchi ${ }^{1}$ and G. Shirleen Roeder ${ }^{1,2,3}$ \\ ${ }^{1}$ Department of Molecular, Cellular, and Developmental Biology, Howard Hughes Medical Institute, Yale University, New \\ Haven, Connecticut 06520, USA; ${ }^{2}$ Department of Genetics, Howard Hughes Medical Institute, Yale University, \\ New Haven, Connecticut 06520, USA
}

In budding yeast, there are two RecA homologs: Rad51 and Dmc1. While Rad51 is involved in both mitotic and meiotic recombination, Dmc1 participates specifically in meiotic recombination. Here, we describe a meiosis-specific protein (Hed1) with a novel Rad51 regulatory function. Several observations indicate that Hed1 attenuates Rad51 activity when Dmc1 is absent. First, although double-strand breaks are normally poorly repaired in the $d m c 1$ mutant, repair becomes efficient when Hed1 is absent, and this effect depends on Rad51. Second, Rad51 and Hed1 colocalize as foci on meiotic chromosomes, and chromosomal localization of Hed1 depends on Rad51. Third, production of Hed1 in vegetative cells inhibits Rad51-dependent recombination events. Fourth, the Hed1 protein shows an interaction with Rad51 in the yeast two-hybrid protein system. We propose that Hed1 provides a mechanism to ensure the coordinated action of Rad51 and Dmc1 during meiosis, by down-regulating Rad51 activity when Dmc1 is unavailable.

[Keywords: Dmc1; Rad51; RecA homologs; crossing over; meiosis; recombination]

Supplemental material is available at http://www.genesdev.org.

Received February 21, 2006; revised version accepted April 25, 2006.

Meiosis plays a central role in sexually reproducing eukaryotes by producing haploid gametes from diploid parental cells (Roeder 1997; Zickler and Kleckner 1999). Unique to meiosis is the reductional division in which homologous chromosomes segregate to opposite poles. Homologous recombination is highly induced during meiotic prophase, and plays two essential roles in segregating homologs at the first meiotic division. First, recombination ensures that each chromosome finds its homologous partner. Second, the subset of recombination events that are resolved as crossovers establish physical connections between homologs that ensure their proper alignment on the spindle apparatus.

Meiotic recombination is initiated with double-strand breaks (DSBs) (Keeney 2001). The ends of DSBs are processed to expose single-stranded DNA tails, which are essential for DNA strand exchange proteins (recombinases) to load onto DSB sites and execute homology searching followed by genetic exchange. Meiotic DSBs are repaired through homologous recombination that occurs predominantly between homologous chromosomes, leading to the formation of either noncrossovers or crossovers (Gerton and Hawley 2005).

${ }^{3}$ Corresponding author.

E-MAIL shirleen.roeder@yale.edu; FAX (203) 432-3263.

Article is online at http://www.genesdev.org/cgi/doi/10.1101/gad.1422506.
As is the case for the majority of eukaryotes, budding yeast has two recombinases, Rad51 and Dmc1, orthologs of the bacterial RecA protein (Bishop et al. 1992; Shinohara et al. 1992). Like RecA, Rad51 and Dmc1 possess homologous DNA pairing and strand exchange activities (Sung 1994; Sehorn and Sung 2004). While Rad51 is essential in both mitotic and meiotic recombination, Dmcl is produced specifically during meiosis, and plays a critical role in meiotic recombination (Bishop and Zickler 2004). In meiotic cells, absence of either of these recombinases results in reduced production of recombinants and the accumulation of recombination intermediates, leading to meiotic cell cycle delay or arrest (Bishop et al. 1992; Shinohara et al. 1992). Simultaneous absence of Rad51 and Dmcl has been shown to reduce crossover formation even further (Dresser et al. 1997; Shinohara et al. 1997), suggesting that Rad51 and Dmc1 have overlapping functions in meiotic recombination.

Although both Rad51 and Dmc1 are important for meiotic recombination, the rad51 mutant phenotype is more severe than that of $d m c 1$ in a number of respects. First, nearly all spores are inviable in the rad51 mutant, whereas some spores ( 20\%) are viable in $d m c 1$ (Game 1983; Rockmill et al. 1995). Second, overproduction of Rad51 almost completely suppresses the meiotic defects of the $d m c 1$ mutant, including the sporulation and crossover defects (Tsubouchi and Roeder 2003). However, 
overproduction of Dmc1 does not suppress rad51 (Tsubouchi and Roeder 2003). Third, Dmc1 fails to localize to chromosomes in the rad51 mutant, but Rad51 is present on chromosomes in the $d m c 1$ mutant (Bishop 1994).

Based on these observations, we have proposed that homology searching and DNA strand exchange can proceed through at least two different pathways during meiosis (Tsubouchi and Roeder 2003). One pathway depends on Rad51, but not Dmc1, and is referred to as the "Rad51-only pathway." The other pathway utilizes both Rad51 and Dmcl, and is referred to as the "Dmc1-dependent pathway." This model explains why some recombination occurs in the dmc1 mutant, and why the meiotic defect of $d m c 1$ is suppressed by overproduction of Rad51. Whether the Rad51-only pathway acts during meiosis in wild-type cells, or only in certain mutant situations, remains to be determined.

Meiotic DSB repair is impaired in the dmc1 mutant, suggesting that Rad51 provides insufficient recombinase activity in meiotic cells, despite the fact that Rad51 functions very efficiently in vegetative cells. Perhaps mitotic and meiotic recombination are mechanistically distinct such that meiotic recombination specifically requires Dmc1, or maybe Rad51 cannot function in the context of meiotic chromosome structure. Our characterization of a novel gene named HED1 sheds light on these issues. Our data suggest that the meiosis-specific Hed1 protein prevents Rad51 from acting except when Dmcl is present.

\section{Results}

Identification of a multicopy suppressor of a RED1 nonnull allele

The RED1 gene encodes a meiosis-specific component of chromosome cores (Smith and Roeder 1997). To generate a nonnull allele of RED1 to use in screening for multicopy suppressors, the gene was randomly mutagenized by a mutagenic PCR. The mutagenized RED1 fragments were cloned, and the resulting plasmids were used to transform a red1-null mutant. Transformants were then screened for the production of viable meiotic progeny (see Materials and Methods). One of the mutants identified, called red1-22, is cold-sensitive for producing viable spores.

After screening a yeast genomic library for genes whose overexpression can suppress the red1-22 defect in viable spore production (see Materials and Methods), one suppressor was identified (Fig. 1A). The plasmid responsible for suppression does not suppress the spore inviability defect of the red1-null mutant (data not shown). Subcloning demonstrated that suppressor activity requires a region containing ORF YDR015c. However, sequencing revealed an extra nucleotide not indicated in the Saccharomyces Genome Database (SGD; http:// www.yeastgenome.org) (Fig. 1B). In light of this change, YDR015c no longer exists as proposed; instead, a new ORF is present on the opposite strand (Fig. 1B). We named this newly identified ORF HED1 (high-copy sup-
A

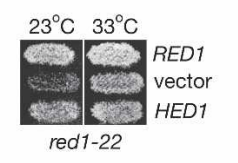

C
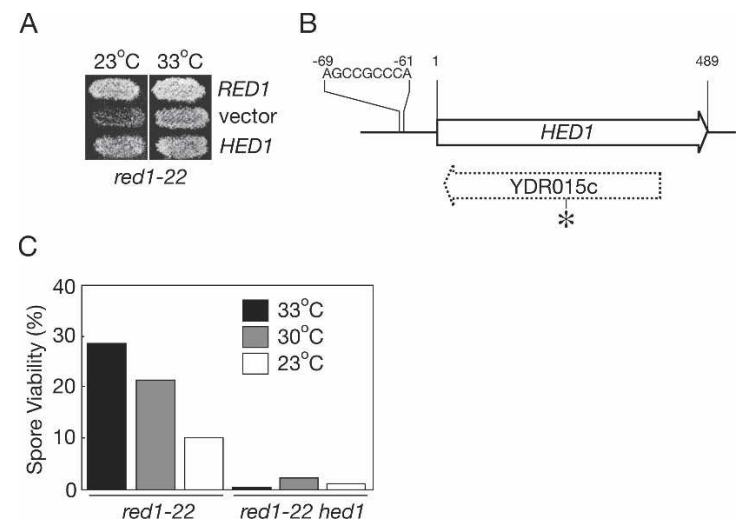

Figure 1. Identification of the HED1 gene. (A) Suppression of the red1 nonnull mutant by Hed1 overproduction. The red1-22 mutant carrying a multicopy vector containing RED1, no insert, or HED 1 was sporulated at permissive $\left(33^{\circ} \mathrm{C}\right)$ and restrictive $\left(23^{\circ} \mathrm{C}\right)$ temperatures and then replica-plated to medium selecting for viable spores (see Materials and Methods). (B) Structure of the HED1 ORF. The upstream sequence that matches well with the URS1 consensus is shown. Numbering starts at the first nucleotide of the first codon for the HED1 ORF. The region corresponding to the original YDR015c is indicated by the dashed arrow. The asterisk indicates the position where an additional nucleotide was found, resulting in earlier termination of the YDR015c ORF and appearance of the HED1 ORF in the opposite strand. (C) Synergistic effect of red1-22 and hed1. Spore viability was tested by tetrad dissection.

pressor of red1). The ORF encodes a protein of 162 amino acids, with no recognizable sequence motifs and no similarity to proteins of known functions. The region upstream of the HED1 gene contains a sequence that matches the URS1 consensus sequence, which confers meiosis-specific gene expression (Mitchell 1994). Consistent with the presence of URS1, HED1 is a member of the early class of meiotic genes, transcribed specifically during meiotic prophase (Chu et al. 1998). Production of the Hed1 protein during early prophase was verified by Western blot analysis (data not shown).

To understand the function of HED1, the gene was disrupted. The hed1 mutant shows wild-type levels of sporulation, but spore viability is slightly decreased $(p<0.05)$ (Fig. 2A,B). However, when hed1 is combined with the red1-22 mutation, almost all spores are inviable at all temperatures tested (Fig. 1C), indicating a synergistic effect between red1 and hed1. Levels of spore formation are similar in red1-22 and red1-22 hed1 strains (data not shown).

\section{Deletion of HED1 improves sporulation in dmc1} and hop2

The Hop2 protein acts in the Dmc1-dependent recombination pathway. Unlike $d m c 1$, chromosomes in hop2 synapse with nonhomologous partners (Leu et al. 1998). Absence of either Dmc1 or Hop2 results in a failure to repair DSBs, causing checkpoint-induced defects in sporulation (Bishop et al. 1992; Rockmill et al. 1995; Leu 
A

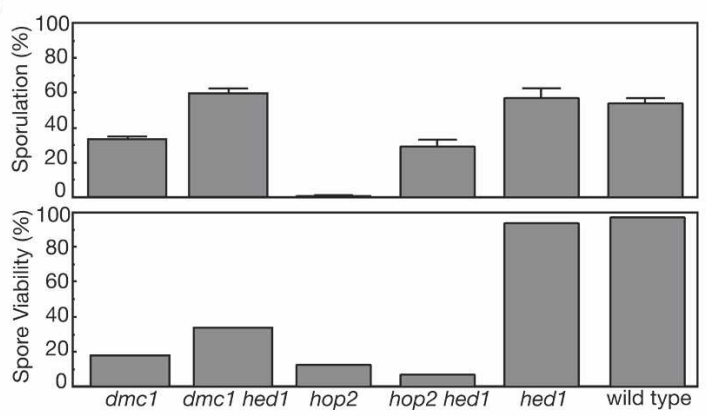

B
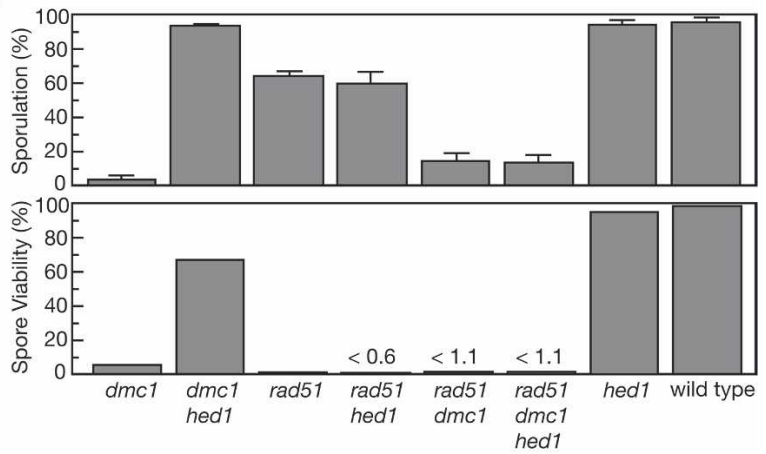

C

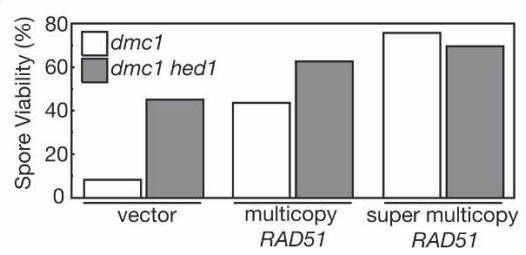

Figure 2. hed1 suppresses the sporulation defect in $d m c 1$ and hop2 and mimics Rad51 overproduction. $(A, B)$ Effect of hed1 on sporulation and spore viability in the BR1919-8B $(A)$ and SK1 $(B)$ strain backgrounds. $(C)$ Effect of Rad51 overproduction in $d m c 1$ and $d m c 1$ hed1 BR1919-8B strains. Cells were sporulated at $30^{\circ} \mathrm{C}$ for $3 \mathrm{~d}(A), 2 \mathrm{~d}(B)$, or $5 \mathrm{~d}(C)$. Error bars represent standard deviations.

et al. 1998). However, if the HED1 gene is deleted in these mutants, sporulation is improved. In the BR19198B diploid strain background (Rockmill and Roeder 1998), the dmc1 hed1 double mutant reaches wild-type levels of sporulation; $30 \%$ of cells sporulate in the hop2 hed1 double mutant (Fig. 2A). The hed1 mutant improves spore viability in the $d m c 1$ mutant, but not in hop2 (Fig. 2A).

In the SK1 strain background (Kane and Roth 1974), the $d m c 1$ mutant shows very strong cell cycle arrest at prophase I (Bishop et al. 1992). Consistent with the results obtained in BR1919-8B, both sporulation and spore viability are greatly improved when the HED1 gene is deleted in $d m c 1$ SK1 strains (Fig. 2B). Interestingly, the hed1 mutation suppresses the spore inviability of $d m c 1$ much better in SK1 ( 60\%) than in BR1919-8B ( 40\%).

The hed1 mutation does not improve sporulation or spore viability when both Rad51 and Dmcl are absent
(Fig. 2B). Thus, hed1 suppression of both defects in $d m c 1$ requires the presence of Rad51.

\section{HED1 deletion mimics the effect of Rad51 overproduction}

Spore inviability of $d m c 1$ is suppressed by overproduction of Rad51 (Tsubouchi and Roeder 2003). To compare the effects of the hed1 mutation and Rad51 overproduction, $d m c 1$ and $d m c 1$ hed1 mutants were transformed with plasmids that achieve three different doses of Rad51. One plasmid is a standard multicopy vector; another is the same vector carrying RAD51 (multicopy RAD51). The third plasmid (super multicopy RAD51) carries both $R A D 51$ and the leu2- $d$ gene, which requires a very high copy number to maintain leucine prototrophy (Conrad et al. 1990).

The hed1 dmc1 double mutant shows a level of spore viability similar to that of the $d m c 1$ mutant carrying multicopy RAD51 (Fig. 2C). Thus, in a dmc1 background, the hed1 mutation phenocopies Rad51 overproduction. The hed1 dmc1 double mutant harboring multicopy RAD51 shows even better spore viability than dmc1 carrying multicopy $R A D 51$, reaching a level similar to that of $d m c 1$ carrying super multicopy RAD51 (Fig. $2 C)$. These results imply that HED 1 has a negative impact on RAD51.

\section{Meiotic DSBs are repaired in dmc1 hed1 and hop2 hed1}

The hed1 mutation suppresses the sporulation defects of both $d m c 1$ and hop2. Since the sporulation defects in these mutants are attributed to a checkpoint mechanism sensing unrepaired DSBs, a logical explanation is that the hed1 mutation allows DSBs to be repaired in $d m c 1$ and hop2. To test this hypothesis, the kinetics of DSB repair on chromosome III were monitored in the BR1919$8 \mathrm{~B}$ strain background. These strains enter in meiosis in a fairly asynchronous manner, making DSBs barely detectable in wild type in which DSBs are formed and concomitantly repaired (data not shown). However, in $d m c 1$ or hop2, DSBs persist at $48 \mathrm{~h}$ after the introduction into sporulation medium (Fig. 3A,B). In the dmc1 hed1 and hop2 hed1 double mutants, a low level of DSBs is detectable at $24 \mathrm{~h}$, but then they disappear, indicating that DSBs are efficiently repaired in the absence of the Hed1 protein (Fig. 3A,B,D). In the dmc1 rad51 hed1 triple mutant, DSBs persist from $20 \mathrm{~h}$ through $48 \mathrm{~h}$ (Fig. 3C,D), indicating that the disappearance of DSBs in the $d m c 1$ hed1 double mutant depends on the presence of Rad51. The extent of the DSB repair defect in the dmc1 rad51 hed1 triple mutant is indistinguishable from that of the dmc1 rad51 double mutant (Fig. 3C,D), providing further evidence that the effect of hed1 in a dmc1 background is dependent upon Rad51.

The kinetics of DSB repair and crossing over (Fig. 4A) were more closely examined in the SK1 strain background, in which cells undergo meiosis in a rapid and 
A
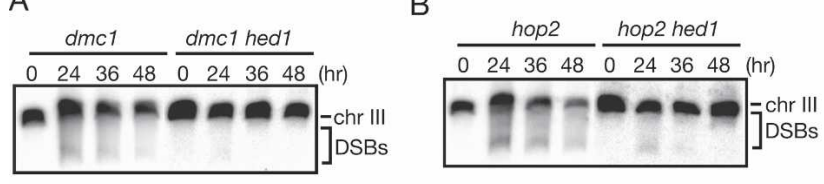

C

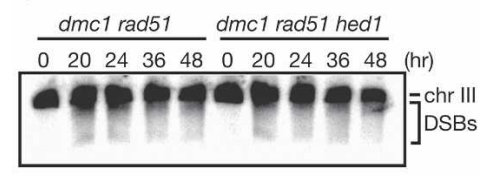

D

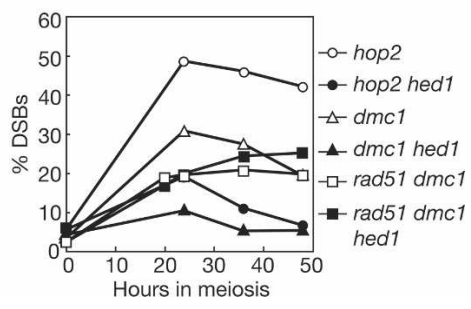

Figure 3. The hed1 mutation improves DSB repair in $d m c 1$ and hop2, but not in rad51 or rad51 dmc1. $(A-C)$ Meiotic DSB repair in $d m c 1, d m c 1$ hed1 $(A)$, hop2, hop2 hed1 $(B)$, and dmc1 rad51, and dmc1 rad51 hed1 $(C)$ BR1919-8B strains. Cells were harvested at the time points indicated. Genomic DNA was subjected to pulsed-field gel electrophoresis followed by Southern blot analysis hybridizing with a probe containing the THR4 gene on chromosome III (chr III). (D) Quantification of DSB products. To calculate the amount of DSB product as a percent of total DNA, the signal strength of the smear below the linear chromosome III band was divided by the sum of the signal strengths of the chromosome III band plus the smear below it. synchronous manner. The hed1 mutant shows kinetics of DSB repair and crossover formation similar to those of wild type (Fig. 4B,C). In the $d m c 1$ mutant, DSBs persist with progressive smearing of bands over time, indicating extensive exonucleolytic digestion of DSB ends (Fig. 4B; Bishop et al. 1992). In the dmc1 hed1 mutant (Fig. 4B,C), unrepaired DSBs do not accumulate. Instead, breaks appear and then disappear as recombinants are formed, although DSB repair is somewhat delayed compared with wild type or hed1 (Fig. 4B,C). The amount of mature recombinants generated in $d m c 1$ hed $1(9.8 \pm 3.3 \%$ of total DNA, mean \pm standard deviation) is higher than in the $d m c 1$ single mutant $(3.2 \pm 0.1 \%)$, but lower than in wild type $(22.8 \pm 0.5 \%)$ or the hed 1 mutant $(19.0 \pm 2.3 \%)$. Consistent with the effects on sporulation and spore viability (Fig. 2B), the hed1 mutation fails to suppress the DSB repair and crossover defects of rad51, and suppression of $d m c 1$ by the hed 1 mutation requires Rad51 (Fig. 4D,E).

\section{Hed1 and Rad51 colocalize to meiotic chromosomes as foci}

To determine the in vivo localization of Hed1, it was fused to green fluorescent protein (GFP) and immunolocalized on spread meiotic nuclei. Cells at different stages of meiotic prophase were identified based on the staining pattern of the Zip1 protein, which is a major component of the synaptonemal complex and serves as a marker for chromosome synapsis. The Hedl protein appears as numerous foci at leptotene (dotty Zip1 staining) and zygotene (dotty and linear Zipl staining), but largely disappears by pachytene (fully linear Zipl staining) (Fig. 5A). The average number of foci at zygotene is $50 \pm 9$ (mean \pm standard deviation) $(n=8)$. This localization pattern is similar to that of the Rad51 and Dmcl proteins (Bishop 1994). Localization of these recombinases to meiotic chromosomes depends on DSB formation catalyzed by the Spo11 protein (Keeney 2001). Hed1 localization also depends on Spol1 (data not shown).

The similarity in immunostaining patterns of Rad51/ Dmc1 and Hed1 prompted us to assess colocalization of these proteins. Nearly all Rad51 foci overlap with Hed1 foci (98\% of Rad51 contain with Hed1; $n=14$ ) (Fig. 5B). Hed1 colocalizes with Dmc1 to a lesser extent $(85 \%$ of Dmc1 foci contain Hed1; $n=11$ ). Hed1 localization to chromosomes is almost completely abolished in the rad51 mutant $(\sim 2.5 \pm 1.7$ foci per spread nucleus, $n=10)$ (Fig. 5C), but neither the pattern nor the timing of Rad51 localization to chromosomes is affected by the absence of Hed1 (data not shown). The dmc1 mutation does not affect the localization of Hed1 to chromosomes (data not shown) or the extent of overlap between Hed1 and Rad51 (94\% of Rad51 foci contain Hed1; $n=27$ ).

Rad51 accumulates and persists on meiotic chromosomes in the hop2 mutant (Tsubouchi and Roeder 2002). Hed1 also shows robust accumulation on meiotic chromosomes in hop2 (118 \pm 40 foci per spread nucleus, $n=12$ ) (Fig. 5D).

Taken together, these observations indicate that Hed1 is recruited to meiotic chromosomes by Rad51 and suggest that Hed1 acts together with Rad51.

\section{Hed1 interacts with Rad51}

The yeast two-hybrid system was used to test for an interaction between Hed1 and Rad51. Full-length proteins were fused to the Gal4-DNA-binding domain (GBD) and to the Gal4 transcription activation domain (GAD), and these fusion plasmids were used to test for interactions between proteins. Hed1 shows a strong interaction with Rad51, in both combinations of plasmids tested (Fig. 5E). In this assay, the strength of the Hed1Rad51 interaction is comparable to that of the Rad51Rad51 interaction. Hed1 also interacts with itself (Fig. 5E).

The ability of Hed1 to interact with Dmcl was also assayed, in an effort to assess the specificity of the Rad51-Hed1 interaction. Hed1 does not interact with Dmcl in the two-hybrid system, though Dmcl interacts strongly with itself, as expected (Fig. 5E; Passy et al. 1999).

\section{Production of Hed1 in vegetative cells inhibits} DSB repair

Our genetic data suggest that Hed1 negatively impacts Rad51, while our cytological and two-hybrid data sug- 
A

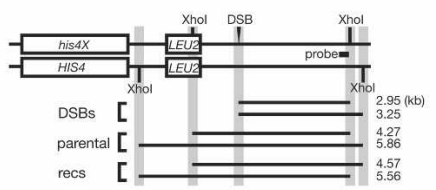

B wild type hed1 $\frac{d m c 1}{0.9 m c 1 \text { hed 1 }}$

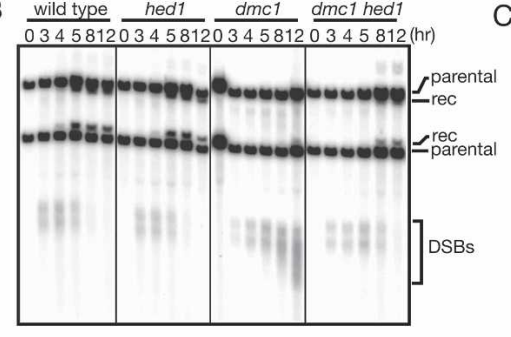

$\mathrm{D}$
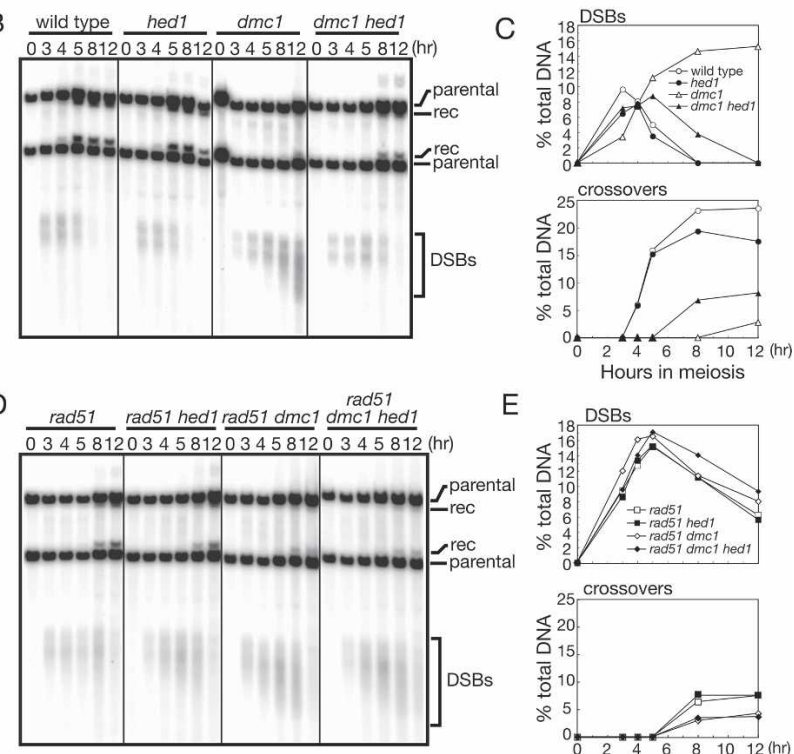

E

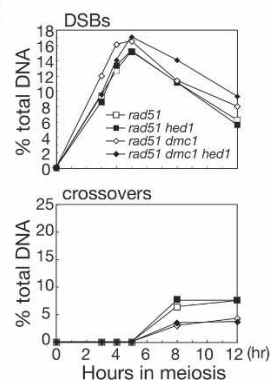

Figure 4. The hed1 mutation increases crossing over in $d m c 1$ but not in rad51 or rad51 dmc1. (A) Structure of the HIS4LEU2 recombination hot spot (Hunter and Kleckner 2001). (B-E) Kinetics of DSB repair and crossing over in wild-type, $d m c 1$, hed1, and $d m c 1$ hed1 $(B, C)$, and rad51, rad51 hed1, rad51 dmc1, and rad51 dmc1 hed1 $(D, E)$ SK1 strains. Genomic DNA was digested with XhoI and subjected to gel electrophoresis followed by Southern blot analysis hybridizing with the probe shown in A. (rec) Recombinant. $(C, E)$ Quantification of DSBs and crossover products. The amount of DSB product (signal below the smaller parental band) and of recombinants were calculated as a percentage of total DNA (sum of the signals corresponding to parental, recombinant, and DSB molecules).

gest that Hed1 and Rad51 directly interact. If Hed1 is an inhibitor of Rad51 activity, then ectopic production of Hed1 during vegetative growth should render cells sensitive to DNA damaging agents that induce DSBs, because homologous recombination is the major pathway for DSB repair. Since Hed1 is a meiosis-specific protein, the HED1 gene was placed under the control of the galactose-inducible promoter (GAL-HED1) to control its expression during vegetative growth; Hed 1 is induced in the presence of galactose and repressed by dextrose.

Remarkably, cells producing Hedl show strong sensitivity to the DNA damaging agent, methylmethane sulfonate (MMS) (Fig. 6A). Cells incubated in the presence of dextrose show MMS resistance similar to wild type, with or without the $G A L-H E D 1$ construct. These results suggest that Hed1, when produced during vegetative growth, interferes with DSB repair. Although cells producing Hed1 generate roughly the same number of colonies as wild type, the colonies are smaller, indicating that Hed1-producing cells grow more slowly than wild type. This is true of many mutants defective in mitotic recombination (e.g., rad52) (Steinmetz et al. 2002).

To confirm that DSB repair is impaired by Hedl, the kinetics of DSBs were monitored. To induce DSBs throughout the genome, cells were treated with $0.1 \%$ MMS for $1 \mathrm{~h}$, then incubated further without MMS. Chromosomes were separated by pulsed-field gel electrophoresis (Fig. 6B). At time zero, prior to exposure to MMS, discrete bands are apparent, each of which represents an intact chromosome. After treating cells with MMS for $1 \mathrm{~h}$, there are no intact chromosomes; instead, there is accumulation of DNA with low molecular weight as a result of DNA breakage. In wild type, broken chromosomes regain their integrity almost completely by $11 \mathrm{~h}$. However, when the Hedl protein is produced, the reappearance of chromosome bands is severely impaired, indicating that Hed1 interferes with DSB repair during vegetative growth.

\section{Hed1 specifically inhibits Rad51-dependent recombination events in vegetative cells}

To determine whether Hed1 production in vegetative cells specifically inhibits Rad51-dependent recombination events, the effect of GAL-HED1 was examined in an assay that measures recombination between tandem repeats of $1 a c Z$, one of which is interrupted by a recognition site for the HO endonuclease (Fig. 6C; Rudin et al. 1989|. Cells also carry the HO endonuclease gene under the control of the galactose promoter; thus, DSBs are formed when cells are transferred into medium containing galactose. In this construct, DSBs are repaired through two pathways: gene conversion (GC) and singlestrand annealing (SSA) (Ivanov et al. 1996). Importantly, the GC pathway requires Rad51, whereas the SSA pathway does not (Ivanov et al. 1996).

Cells were incubated in the presence of galactose for 7 $\mathrm{h}$, and genomic DNA was then analyzed by Southern blotting. In wild type, both GC and SSA products are detected, with SSA products being considerably more abundant $(49 \pm 13 \%$ of total DNA, mean \pm standard deviation) than GC products $(16 \pm 4 \%)$ (Fig. $6 \mathrm{D}$, left). In cells producing Hed1, GC is greatly reduced $(2 \pm 1 \%)$, while SSA appears to be slightly increased $(60 \pm 19 \%)$ (Fig. 6D, right). Thus, in this assay, Hed1 specifically inhibits Rad51-dependent recombination events.

\section{Discussion}

Hed1 is a meiosis-specific protein that down-regulates Rad51-mediated recombination when the meiotic recombination machinery is impaired

Meiotic recombination plays two essential roles during meiosis: to facilitate recognition between homologous chromosomes, and to establish physical connections between homologs through crossing over. Unlike mitotic recombination, meiotic recombination relies largely on 
A wild type

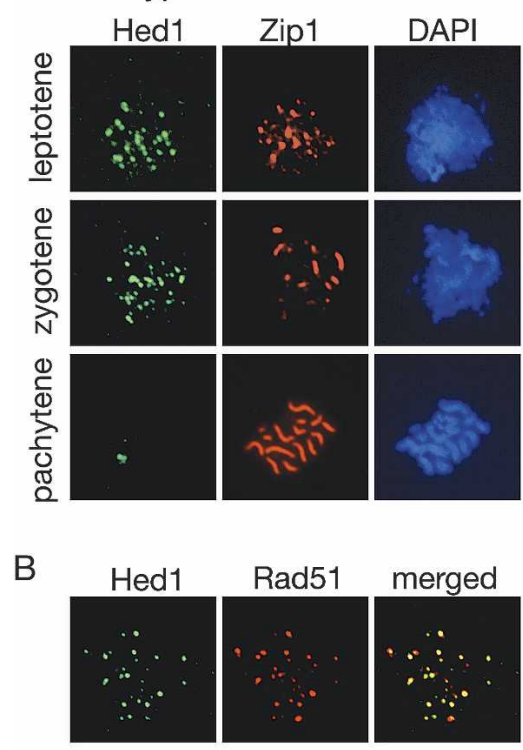

C rad51

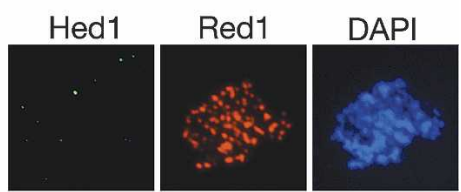

D hop2 Hed1

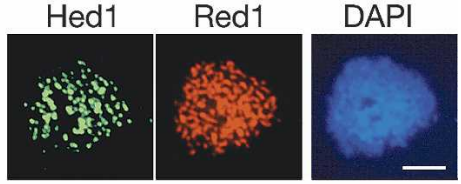

E

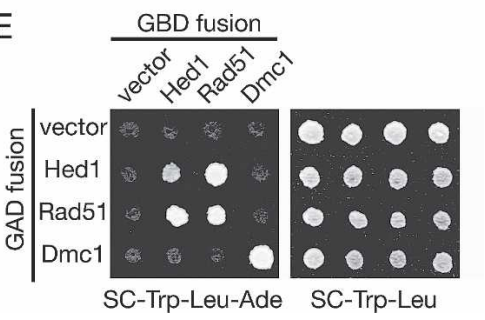

Figure 5. Hed1 colocalizes and interacts with Rad51. (A) Hed1 localizes to foci on chromosomes early in meiotic prophase. Meiotic chromosomes from wild-type cells producing GFPtagged Hed1 were stained with anti-GFP and anti-Zip1 antibodies, and with the DNA-binding dye, 4',6'-diamino-2-phenylindole (DAPI). Hedl-GFP is estimated to be functional to $80 \%$ of the wild-type level (see Materials and Methods). (B) Colocalization of Hed1 and Rad51. Nuclei from cells producing GFPtagged Hedl were stained with anti-GFP and anti-Rad51 antibodies. (C) Hed1 localization depends on Rad51. Meiotic chromosomes from rad51 cells producing GFP-tagged Hed1 were stained with anti-GFP and anti-Red 1 antibodies, and with DAPI. (D) Hed1 accumulates on chromosomes in hop2 strains. Meiotic chromosomes from hop2 cells producing GFPtagged Hed1 were stained with anti-GFP and anti-Red 1 antibodies, and with DAPI. Red 1 is a component of the core of meiotic chromosomes and was used to identify cells at prophase I. Cytological experiments were carried out in the BR1919-8B strain background. Bar, $4 \mu \mathrm{m}$. (E) Hed1 interacts with Rad51 in the two-hybrid protein assay. Medium lacking tryptophan and leucine was used to maintain the GBD fusion plasmid (marked with TRP1) and the GAD fusion plasmid (marked with LEU2). Growth on medium lacking adenine (shown on the left) reflects the expression level of the GAL4-ADE2 reporter gene and is thus a measure of the interaction between two fusion proteins. Shown on the right is growth on medium lacking tryptophan and leucine as a reference.

Dmc1, a meiosis-specific recombinase, suggesting that Rad51 activity is somehow compromised in meiotic cells. The Dmc1 dependency of meiotic recombination is suppressed by mutations in genes encoding components of meiotic chromosome cores (Xu et al. 1997; Wan et al. 2004), suggesting a close relationship between meiotic chromosome structure and the requirement for Dmc1. Here, we present the surprising discovery of a meiotic protein that alleviates the requirement for Dmc1 without affecting the structure of meiotic chromosomes.

Several lines of evidence indicate that the Hed1 protein inhibits Rad51. First, loss of Hed1 mimics overproduction of Rad51. Second, production of Hed1 in vegetative cells renders them deficient in DSB repair by specifically inhibiting Rad51-dependent recombination events. Third, Hed1 and Rad51 colocalize on meiotic chromosomes, and Hed1 interacts with Rad51 in the two-hybrid protein system. Finally, the absence of Hed1 allows DSBs to be repaired efficiently in the dmc1 and hop2 mutants, and this repair depends on Rad51. Inhibition of Rad51 by the wild-type Hed1 protein can explain the inefficiency of meiotic DSB repair under conditions where the Dmc1-dependent pathway is inactivated (e.g., by a dmc1 or hop2 mutation).

A number of observations suggest that Hed1 does not inhibit Dmc1. The rad51 and rad51 dmc1 defects in sporulation and recombination are not suppressed in the absence of Hed1. Furthermore, Hed1 does not interact with Dmcl in the two-hybrid system. Taken together, these results indicate that Hedl acts specifically on Rad51.
Crossing over is reduced when Rad51 is the only recombinase

Identification of a meiosis-specific inhibitor of Rad51 has allowed us to examine the meiotic function of Rad51 in the absence of Dmc1, under conditions where DSBs are formed at the wild-type level and morphogenesis of the synaptonemal complex proceeds normally. In $d m c 1$ hed1 strains, meiotic DSBs are repaired efficiently and crossovers are formed at a higher level than in the $d m c 1$ single mutant, but to a lesser extent than in wild type. These results indicate that Rad51 is sufficient for repairing meiotic DSBs, but not sufficient to support wild-type levels of crossing over.

Why is Dmcl required to achieve the levels of crossing over observed in wild-type cells? One possibility is that Dmc1 preferentially facilitates recombination between nonsister chromatids as opposed to exchange between sister chromatids (Schwacha and Kleckner 1997). In meiotic cells, recombination events between nonsister chromatids exceed exchanges between sisters by threefold to 10-fold (Petes and Pukkila 1995). Alternatively, Dmc1 might promote crossovers at the expense of noncrossovers. Unlike mitotic recombination, in which DSBs are mostly repaired as noncrossovers, a significant fraction $(\sim 30 \%)$ of meiotic recombination events are associated with crossing over (Fogel et al. 1981).

Regardless of whether Dmcl promotes exchange between nonsisters (versus sisters) or crossovers (versus noncrossovers), it is not absolutely required for the enrichments normally observed in meiotic cells. In both dmc1 hed1 strains and in $d m c 1$ strains overproducing Rad51, spore viability is fairly high and interhomolog 
A

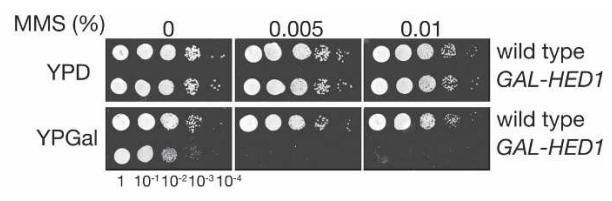

B

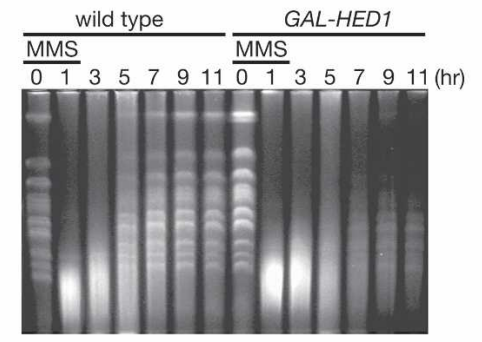

C

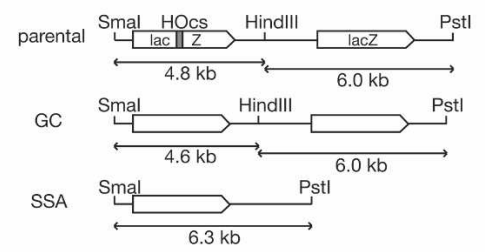

D

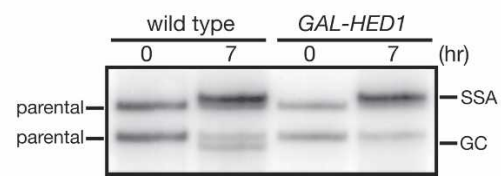

Figure 6. Production of Hed1 inhibits DSB repair in vegetatively growing cells. $(A)$ Hed1 production renders vegetative cells sensitive to MMS. Suspensions of wild-type cells and cells carrying the HED1 gene fused to the galactose-inducible promoter (GAL-HED1) were serially diluted (10-fold dilutions) and then placed on complete medium containing either dextrose (YPD) or galactose (YPGal). Plates contain various concentrations of MMS as indicated. The hed1-null mutant shows MMS resistance similar to wild type on both dextrose- and galactosecontaining media (data not shown). (B) Hed1 inhibits DSB repair in vegetative cells. Wild-type cells and cells with the GAL$H E D 1$ construct were preincubated in the presence of galactose. Cells preincubated in the presence of galactose were treated with $0.1 \%$ MMS for $1 \mathrm{~h}$; the MMS was then washed away and cells were incubated in the presence of galactose. Samples were harvested at the time points indicated. Genomic DNA was subjected to pulsed-field gel electrophoresis and DNA was stained with ethidium bromide. $(C)$ Structure of the lac $Z$ tandem repeats used to detect DSB repair. The gray box represents two copies of the HO-endonuclease recognition site (HOcs). A DSB formed by the $\mathrm{HO}$ endonuclease in the lac $Z$ repeat on the left is repaired through two pathways, GC and SSA. Each pathway produces a specific set of DNA fragments after digestion with SmaI, HindIII, and PstI. (D) Hed1 inhibits GC but not SSA. Wild-type cells and cells with the GAL-HED1 construct, both carrying the galactose-inducible $\mathrm{HO}$ endonuclease gene and the tandem-repeat construct, were incubated in medium containing galactose. Genomic DNA was extracted from samples harvested at 0 and $7 \mathrm{~h}$. GC and SSA products were detected by Southern blot hybridization using a probe that hybridizes to the lacZ gene (see Materials and Methods).

crossovers are frequent. Thus, a significant fraction of exchange events occur between nonsister chromatids in these strain backgrounds, though the magnitude of this bias might not be as pronounced as in wild-type cells. Similarly, a significant fraction of DSBs must be repaired as crossovers, though the fraction of repair events associated with crossing over may be reduced compared with wild type.

This discussion suggests a possible mechanism for suppression of the red1-22 mutation by Hedl overproduction, and for the synergistic effects of the red1-22 and hed1 mutations. Previous studies have indicated that Red1 plays a role in preventing recombination between sister chromatids (Schwacha and Kleckner 1997); thus, the interhomolog bias might well be reduced in the red122 mutant. Deletion of HED1 exacerbates the red1-22 defect presumably by up-regulating the Rad51-only pathway, which further contributes to the defect in interhomolog crossing over. Conversely, Hed1 overproduction suppresses this defect, possibly by shutting down the Rad51-only pathway and thereby enhancing the activity of the Dmc1-dependent pathway, which is more effective in promoting crossovers between homologs. Hed1 overproduction does not suppress the red1-null mutation, demonstrating that Hedl overproduction cannot fully compensate for the absence of Red1.

\section{Hed1 may coordinate Rad51 and Dmc1}

Cells may need to prevent Rad51 from acting alone in order to optimize interhomolog crossover frequencies. Indeed, the majority of Rad51 foci on meiotic chromosomes are found colocalized with Dmc1 (Bishop 1994). Furthermore, in the absence of Dmc1, meiotic recombination is severely delayed, and this delay is reduced in the $d m c 1$ hed1 double mutant. Thus, Hed1 appears to provide a mechanism to prevent Rad51 from acting alone, thereby ensuring that most DSBs are processed through the combined action of Rad51 and Dmc1.

The observation that a majority of Rad51 foci contain Dmc1, and vice versa, suggests that most recombination events involve both proteins. However, almost all Rad51 foci also contain Hedl. Thus, Hedl is associated with Rad51 at all times, but may inhibit Rad51 only when Dmcl is absent; for instance, Dmcl might promote a conformational change in the Hed1-Rad51 complex that relieves inhibition without releasing the Hed 1 protein. It is also possible that the presence of Rad51, but not its activity, is important for Dmcl to act on DSBs. In this case, Rad51 may remain enzymatically inactive throughout meiosis.

The modest phenotype of the hed1 single mutant (slight reduction in spore viability) suggests that Rad51 and Dmcl normally act in a fairly coordinate fashion even when Hedl is absent. In a wild-type strain background, Hedl might function only in a subset of nuclei or at a subset of recombinational interactions. Hedl may provide a quality control mechanism to hold Rad51 activity in check when the amount of Dmc1 is insufficient to support the coordinated action of Dmc1 and Rad51. In nature, induction of DMC1 might not be as robust as it is under laboratory conditions. In the lab, cells are abruptly deprived of carbon and nitrogen sources, 
whereas starvation may often be more gradual under natural conditions (Honigberg and Purnapatre 2003). Also, even after entry into meiosis, yeast cells are capable of switching back to vegetative growth, during which Dmc1 is no longer necessary for homologous recombination (Schwacha and Kleckner 1997; Zenvirth et al. 1997). Thus, in nature, Hed1 might play a critical role in inhibiting meiotic recombination either until enough Dmcl has accumulated to support the coordinated action of Rad51 and Dmc1 or until nutrients are restored and vegetative growth is resumed.

Schwacha and Kleckner (1997) have previously proposed that Red1 functions similarly to Hed1 based on the observation that a red1 mutation suppresses the $d m c 1$ defects in DSB repair and sporulation (red1 also reduces DSBs to $\sim 25 \%$ of the wild-type level). Perhaps Hed1 and Red1 act together to inhibit the action of Rad51 until Dmcl is loaded onto chromosomes. An alternative interpretation is that red1 acts indirectly to relieve the requirement for Dmc1 by modifying chromosome structure. The red1 mutant fails to make synaptonemal complex; sister-chromatid cohesion is defective (Bailis and Roeder 1998), and the cores of meiotic chromosomes (axial elements) fail to develop properly (Rockmill and Roeder 1990). Thus, in important respects, chromosomes present in the red1 mutant may resemble the chromosomes present in vegetative cells, where there is no impediment to Rad51 activity.

A number of observations suggest that Red1 and Hed1 exert their effects on Rad51 through different mechanisms, with Hed1 acting more directly. First, the Hed1 protein shows near perfect colocalization with Rad51, whereas Red1 localizes in linear stretches along chromosomes. Second, the hed1 dmc1 mutant produces viable spores, but the red1 dmc1 mutant produces inviable products. Third, overproduction of Hed1 interferes with DSB repair during vegetative growth when the Red1 protein is not present. The phenotype of the hed1 mutant, and its similarity to Rad51 overproduction, suggest that Hed1 functions exclusively in regulating Rad51 activity. In contrast, the pleiotropic phenotype of the red1 mutant indicates that Red1 has a variety of functions, presumably reflecting its role as a basic building block of meiotic chromosomes.

\section{Attenuators of homologous recombination}

Several proteins are known to attenuate homologous recombination. The Saccharomyces cerevisiae Srs2 protein is an antirecombinase that acts to prevent inappropriate recombination events in mitotic cells (Krejci et al. 2003; Veaute et al. 2003). Srs2 has a DNA helicase activity and antagonizes Rad51 by disrupting the Rad51 presynaptic filament. More recently, another helicase, Schizosaccharmyces pombe Fbh1, also has been implicated in destabilizing the Rad51 presynaptic filament (Morishita et al. 2005; Osman et al. 2005). The absence of an apparent helicase motif in Hed1 implies that Hed1 inhibits homologous recombination by a different mechanism. One possibility is suggested by the behavior of the Escherichia coli RecX protein, which attenuates RecA-dependent recombination reactions. RecX does not possess a helicase motif, and it inhibits RecA by blocking extension of the RecA presynaptic filament (Drees et al. 2004). It will therefore be of interest to determine whether Hed1 affects Rad51 presynaptic filament growth on single-stranded DNA.

\section{Is the mechanism provided by Hed1 conserved in higher eukaryotes?}

So far, homology searches have not identified a Hed1 ortholog in other organisms, aside from budding yeast species. However, mouse cells lacking Dmcl show strong cell cycle arrest in meiotic prophase, with persistent accumulation of Rad51 foci on meiotic chromosomes (Pittman et al. 1998; Yoshida et al. 1998). This phenotype is similar to that observed in the budding yeast $d m c 1$ mutant, and is therefore consistent with the possibility that a Hed1-like activity exists in mammalian cells.

\section{Hed1 as a molecular tool}

The ability of Hed1 to inhibit recombination in vegetative cells indicates that Hed1 can function in the absence of any other meiosis-specific proteins. Thus, Hed1 production could provide a means to inhibit recombination in cell types in which genes encoding recombination enzymes cannot easily be knocked out. Hed1 may therefore prove to be a valuable tool in the molecular biologist's toolbox.

\section{Materials and methods}

\section{Yeast strains}

Genotypes of yeast strains are given in Supplementary Table 1. Strain construction and strains used in each study are indicated in the Supplemental Material. The sequence corresponding to YDR015c was replaced with KAN to create hed1::KAN (Longtine et al. 1998). The sequence corresponding to HED1 was replaced with HYG to create hed1::HYG /Goldstein and McCusker 1999).

The sequence encoding GFP was integrated at the $3^{\prime}$ end of the HED1 gene to generate HED1-GFP using the GFP-KAN cassette (Longtine et al. 1998). Since the hed1-null mutant by itself does not have detectable phenotypes, the functionality of the HED1-GFP fusion construct was tested in a hop2 mutant background. Spore formation in the hop 2 and hop 2 hed1 mutants is $1.3 \%$ and $26.1 \%$, respectively. HED1-GFP in a hop2 mutant background showed $6.2 \%$ sporulation, indicating that HED1-GFP is functional to $80 \%$ of the level of the wild-type HED1 with respect to sporulation. The sequence upstream of the HED 1 gene ( -77 to -1 , the first nucleotide of the first codon as 1) was replaced by the galactose-inducible promoter using the KAN-GAL1 cassette (Longtine et al. 1998) to create GAL-HED1.

pJH273 is a centromere-based plasmid marked with LEU2 and $U R A 3$ and carrying tandem repeats of lacZ. One copy of lac $Z$ is under the control of the CYC1 promoter and interrupted by two copies of the recognition site for the $\mathrm{HO}$ endonuclease; the other copy of lacZ lacks a promoter (Rudin et al. 1989) (see also Fig. 5C).

For yeast two-hybrid protein analyses, PCR-amplified HED1, RAD51, and DMC1 were cloned between the PvuII and NcoI 
sites of the pOAD and pOBD2 plasmids (Hudson et al. 1997). For $D M C 1$, cDNA was used as a template (Hong et al. 2001). GAL sequences were fused to the $5^{\prime}$ end of each ORF.

\section{Isolating multicopy suppressors of the red1 mutant}

The DNA fragment carrying the RED1 gene was amplified by PCR, and PCR products were ligated to YCplac22 (Gietz and Sugino 1988) digested with HindIII and EcoRI to generate a pool of randomly mutagenized RED1 genes. The pool was then used to transform a red1 trp1 yeast strain, and $\operatorname{Trp}^{+}$colonies were selected. These transformants were screened by replica plating onto sporulation medium, incubating at different temperatures $\left(23^{\circ} \mathrm{C}\right.$ and $\left.33^{\circ} \mathrm{C}\right)$, and then replica plating onto medium containing canavanine and cycloheximide to select for viable spores (Tsubouchi and Roeder 2002). One mutant, red1-22, shows a more pronounced reduction in spore viability at $23^{\circ} \mathrm{C}$ than at $33^{\circ} \mathrm{C}$. Yeast cells carrying the red1-22 allele on a TRP1-marked plasmid were transformed with a yeast genomic library in YEp24, and $\mathrm{Trp}^{+} \mathrm{Ura}^{+}$transformants were selected (Botstein et al. 1979; Carlson and Botstein 1982). These transformants were sporulated at $23^{\circ} \mathrm{C}$ and then replica plated to medium containing canavanine and cycloheximide to screen for clones that display increased spore viability at $23^{\circ} \mathrm{C}$.

\section{Analysis of DSB repair kinetics after MMS treatment}

A strain carrying $G A L-H E D 1$ or a control vector were used to examine DSB repair after exposure to MMS. Single colonies were inoculated into $2 \mathrm{~mL}$ of YPD and incubated for $12 \mathrm{~h}$ at $30^{\circ} \mathrm{C}$. These cultures were transferred to $25 \mathrm{~mL}$ of YPD and incubated for another $12 \mathrm{~h}$. Cells were then washed and resuspended into YPGlycerol (1\% yeast extract, $2 \%$ bactopeptone, $2 \%$ glyceroll and incubated for $12 \mathrm{~h}$. Cells were then resuspended in $50 \mathrm{~mL}$ of YPGalactose $(1 \%$ yeast extract, $2 \%$ bactopeptone, $2 \%$ galactose) for $1 \mathrm{~h}$. The end of this $1-\mathrm{h}$ incubation is indicated as the 0 -h time point (Fig. 5B). MMS was added to a final concentration of $0.1 \%$, and cultures were incubated for $1 \mathrm{~h}$. MMS was then inactivated by treatment with sodium thiosulfate; cells were washed with water once and resuspended in 45 $\mathrm{mL}$ of YPGalactose. Five milliliters of cell culture were harvested at each of the indicated time points (Fig. 5B). Genomic DNA was subjected to pulsed-field gel electrophoresis followed by ethidium bromide staining to visualize DNA.

\section{Detection of SSA and GC}

Single colonies from strains carrying lac $Z$ repeats were inoculated into $2 \mathrm{~mL}$ of SC-Ura and incubated overnight at $30^{\circ} \mathrm{C}$. Cells were then spun down and resuspended in $25 \mathrm{~mL}$ of SC-Ura and incubated for $24 \mathrm{~h}$. Cells were then washed once with water, resuspended in $50 \mathrm{~mL}$ of YPGlycerol, and incubated for 11 h. Cells were then spun down and resuspended in YPGalactose, and $5 \mathrm{~mL}$ of the culture was immediately taken and used for the 0 -h time point (Fig. 5D). Another sample was taken at $7 \mathrm{~h}$. DNA was extracted and digested with HindIII, PstI, and SmaI. The fragments corresponding to the products of SSA and GC, were visualized by Southern blot analysis hybridizing with a probe derived from the lac $Z$ ORF fragment. To calculate the percentage of DNA present as GC or SSA products, the signal strength of the band corresponding to each product was divided by the sum of the signal strengths of all four bands (GC, SSA, and the two parental fragments) (Fig. 5D).

\section{Other techniques}

Detection of meiotic DSBs in BR1919-8B and in SK1 was described previously (Schwacha and Kleckner 1997; Tsubouchi and Roeder 2003). Chromosome spreads and immunostaining were carried out as described previously (Tsubouchi and Roeder 2002). Spore formation and viability were measured as described previously (Tsubouchi and Roeder 2004). At least 44 tetrads were dissected to measure spore viability.

\section{Acknowledgments}

We thank Douglas Bishop, Jim Haber, Neil Hunter, and Nancy Kleckner for providing plasmids, yeast strains, and antibodies. We are grateful to members of the Roeder Lab for useful comments on the manuscript. H.T. especially thanks Allison Adonizio for technical assistance, and Patrick Sung, Michael Sehorn, and Tomomi Tsubouchi for stimulating discussions. This work was supported by the Howard Hughes Medical Institute and by National Institutes of Health grant GM28904.

\section{References}

Bailis, J.M. and Roeder, G.S. 1998. Synaptonemal complex morphogenesis and sister-chromatid cohesion require Mek1-dependent phosphorylation of a meiotic chromosomal protein. Genes \& Dev. 12: 3551-3563.

Bishop, D.K. 1994. RecA homologs Dmc1 and Rad51 interact to form multiple nuclear complexes prior to meiotic chromosome synapsis. Cell 79: 1081-1092.

Bishop, D.K. and Zickler, D. 2004. Early decision: Meiotic crossover interference prior to stable strand exchange and synapsis. Cell 117: 9-15.

Bishop, D.K., Park, D., Xu, L., and Kleckner, N. 1992. DMC1: A meiosis-specific yeast homolog of $E$. coli recA required for recombination, synaptonemal complex formation, and cell cycle progression. Cell 69: 439-456.

Botstein, D., Falco, S.C., Stewart, S.E., Brennan, M., Scherer, S., Stinchcomb, D.T., Struhl, K., and Davis, R.W. 1979. Sterile host yeast (SHY): A eukaryotic system of biological containment for recombinant DNA experiments. Gene 8: 17-24.

Carlson, M. and Botstein, D. 1982. Two differentially regulated mRNAs with different $5^{\prime}$ ends encode secreted and intracellular forms of yeast invertase. Cell 28: 145-154.

Chu, S., DeRisi, J., Eisen, M., Mulholland, J., Botstein, D., Brown, P.O., and Herskowitz, I. 1998. The transcriptional program of sporulation in budding yeast. Science 282: 699703.

Conrad, M.N., Wright, J.H., Wolf, A.J., and Zakian, V.A. 1990. RAP1 protein interacts with yeast telomeres in vivo: Overproduction alters telomere structure and decreases chromosome stability. Cell 63: 739-750.

Drees, J.C., Lusetti, S.L., Chitteni-Pattu, S., Inman, R.B., and Cox, M.M. 2004. A RecA filament capping mechanism for RecX protein. Mol. Cell 15: 789-798.

Dresser, M., Ewing, D., Conrad, M., Dominguez, A., Barstead, R., Jiang, H., and Kodadek, T. 1997. DMC1 functions in a meiotic pathway that is largely independent of the RAD51 pathway. Genetics 147: 533-544.

Fogel, S., Mortimer, R.K., and Lusnak, K. 1981. Mechanisms of meiotic gene conversion, or 'wanderings on a foreign strand.' In The molecular biology of the yeast Saccharomyces: Life cycle and inheritance (eds. J.N. Strathern et al.), pp. 289-339. Cold Spring Harbor Laboratory Press, Cold Spring Harbor, NY.

Game, J.C. 1983. Radiation-sensitive mutants and repair in yeast. In Yeast genetics (eds. J.F.T. Spencer et al.), pp. 109137. Springer-Verlag, New York.

Gerton, J.L. and Hawley, R.S. 2005. Homologous chromosome 
interactions in meiosis: Diversity amidst conservation. Nat. Rev. Genet. 6: 477-487.

Gietz, R.D. and Sugino, A. 1988. New yeast-Escherichia coli shuttle vectors constructed with in vitro mutagenized yeast genes lacking six-base pair restriction sites. Gene 74: 527-534.

Goldstein, A.L. and McCusker, J.H. 1999. Three new dominant drug resistance cassettes for gene disruption in Saccharomyces cerevisiae. Yeast 15: 1541-1553.

Hong, E.L., Shinohara, A., and Bishop, D.K. 2001. Saccharomyces cerevisiae Dmcl protein promotes renaturation of single-strand DNA (ssDNA) and assimilation of ssDNA into homologous super-coiled duplex DNA. J. Biol. Chem. 276: 41906-41912.

Honigberg, S.M. and Purnapatre, K. 2003. Signal pathway integration in the switch from the mitotic cell cycle to meiosis in yeast. J. Cell Sci. 116: 2137-2147.

Hudson Jr., J.R., Dawson, E.P., Rushing, K.L., Jackson, C.H., Lockshon, D., Conover, D., Lanciault, C., Harris J.R., Simmons, S.J., Rothstein, R., et al. 1997. The complete set of predicted genes from Saccharomyces cerevisiae in a readily usable form. Genome Res. 7: 1169-1173.

Hunter, N. and Kleckner, N. 2001. The single-end invasion: An asymmetric intermediate at the double-strand break to double-Holliday junction transition of meiotic recombination. Cell 106: 59-70.

Ivanov, E.L., Sugawara, N., Fishman-Lobell, J., and Haber, J.E. 1996. Genetic requirements for the single-strand annealing pathway of double-strand break repair in Saccharomyces cerevisiae. Genetics 142: 693-704.

Kane, S.M. and Roth, R. 1974. Carbohydrate metabolism during ascospore development in yeast. J. Bacteriol. 118: 8-14.

Keeney, S. 2001. Mechanism and control of meiotic recombination initiation. Curr. Top. Dev. Biol. 51: 1-53.

Krejci, L., Van Komen, S., Li, Y., Villemain, J., Reddy, M.S., Klein, H., Ellenberger, T., and Sung, P. 2003. DNA helicase Srs2 disrupts the Rad51 presynaptic filament. Nature 423: 305-309.

Leu, J.-Y., Chua, P.R., and Roeder, G.S. 1998. The meiosis-specific Hop2 protein of $S$. cerevisiae ensures synapsis between homologous chromosomes. Cell 94: 375-386.

Longtine III, M.S., Demarini, D.J., Shah, N.G., Wach, A., Brachat, A., Phillippsen, P., and Pringle, J.R. 1998. Additional modules for versatile and economical PCR-based gene deletion and modification in Saccharomyces cerevisiae. Yeast 14: $953-961$.

Mitchell, A.P. 1994. Control of meiotic gene expression in Saccharomyces cerevisiae. Microbiol. Rev. 58: 56-70.

Morishita, T., Furukawa, F., Sakaguchi, C., Toda, T., Carr, A.M., Iwasaki, H., and Shinagawa, H. 2005. Role of the Schizosaccharomyces pombe F-Box DNA helicase in processing recombination intermediates. Mol. Cell. Biol. 25: 8074-8083.

Osman, F., Dixon, J., Barr, A.R., and Whitby, M.C. 2005. The F-Box DNA helicase Fbh1 prevents Rhp51-dependent recombination without mediator proteins. Mol. Cell. Biol. 25: 8084-8096.

Passy, S.I., Yu, X., Li, Z., Radding, C.M., Masson, J.-Y., West, S.C., and Egelman, E.H. 1999. Human Dmc1 protein binds DNA as an octameric ring. Proc. Natl. Acad. Sci. 96: 1068410688.

Petes, T.D. and Pukkila, P.J. 1995. Meiotic sister chromatid recombination. Adv. Genet. 33: 41-62.

Pittman, D.L., Cobb, J., Schimenti, K.J., Wilson, L.A., Cooper, D.M., Brignull, E., Brignull, M., Handel, M.A., and Schimenti, J.C. 1998. Meiotic prophase arrest with failure of chromosome synapsis in mice deficient for Dmc1, a germ- line-specific RecA homolog. Mol. Cell 1: 697-705.

Rockmill, B. and Roeder, G.S. 1990. Meiosis in asynaptic yeast. Genetics 126: 563-574.

. 1998. Telomere-mediated chromosome pairing during meiosis in budding yeast. Genes \& Dev. 12: 2574-2586.

Rockmill, B., Sym, M., Scherthan, H., and Roeder, G.S. 1995. Roles for two RecA homologs in promoting meiotic chromosome synapsis. Genes \& Dev. 9: 2684-2695.

Roeder, G.S. 1997. Meiotic chromosomes: It takes two to tango. Genes \& Dev. 11: 2600-2621.

Rudin, N., Sugarman, E., and Haber, J.E. 1989. Genetic and physical analysis of double-strand break repair and recombination in Saccharomyces cerevisiae. Genetics 122: 519-534.

Schwacha, A. and Kleckner, N. 1997. Interhomolog bias during meiotic recombination: Meiotic functions promote a highly differentiated interhomolog-only pathway. Cell 90: 11231136.

Sehorn, M.G. and Sung, P. 2004. Meiotic recombination-An affair of two recombinases. Cell Cycle 3: 1375-1377.

Shinohara, A., Ogawa, H., and Ogawa, T. 1992. Rad51 protein involved in repair and recombination in $S$. cerevisiae is a RecA-like protein. Cell 69: 457-470.

Shinohara, A., Gasior, S., Ogawa, T., Kleckner, N., and Bishop, D.K. 1997. Saccharomyces cerevisiae recA homologues RAD51 and DMC1 have both distinct and overlapping roles in meiotic recombination. Genes Cells 2: 615-629.

Smith, A.V. and Roeder, G.S. 1997. The yeast Red1 protein localizes to the cores of meiotic chromosomes. J. Cell Biol. 136: $957-967$.

Steinmetz, L.M., Scharfe, C., Deutschbauer, A.M., Mokranjac, D., Herman, Z.S., Jones, T., Chu, A.M., Giaever, G., Prokisch, H., Oefner, P.J., et al. 2002. Systematic screen for human disease genes in yeast. Nat. Genet. 31: 400-404.

Sung, P. 1994. Catalysis of ATP-dependent homologous DNA pairing and strand exchange by yeast Rad51 protein. Science 265: 1241-1243.

Tsubouchi, H. and Roeder, G.S. 2002. The Mnd1 protein forms a complex with Hop2 to promote homologous chromosome pairing and meiotic double-strand break repair. Mol. Cell. Biol. 22: 3078-3088.

- 2003. The importance of genetic recombination for fidelity of chromosome pairing in meiosis. Dev. Cell 5: 915-925.

- 2004. The budding yeast Mei5 and Sae3 proteins act together with Dmcl during meiotic recombination. Genetics 168: 1219-1230.

Veaute, X., Jeusset, J., Soustelle, C., Kowalczykowski, S.C., Le Cam, E., and Fabre, F. 2003. The Srs2 helicase prevents recombination by disrupting Rad51 nucleoprotein filaments. Nature 423: 309-312.

Wan, L.H., de los Santos, T., Zhang, C., Shokat, K., and Hollingsworth, N.M. 2004. Mek1 kinase activity functions downstream of RED1 in the regulation of meiotic double strand break repair in budding yeast. Mol. Biol. Cell 15: 11-23.

$\mathrm{Xu}$, L., Weiner, B.M., and Kleckner, N. 1997. Meiotic cells monitor the status of the interhomolog recombination complex. Genes \& Dev. 11: 106-118.

Yoshida, K., Kondoh, G., Matsuda, Y., Habu, T., Nishimune, Y., and Morita, T. 1998. The mouse RecA-like gene Dmc1 is required for homologous chromosome synapsis during meiosis. Mol. Cell 1: 707-718.

Zenvirth, D., Loidl, J., Klein, S., Arbel, A., Shemesh, R., and Simchen, G. 1997. Switching yeast from meiosis to mitosis: Double-strand break repair, recombination and synaptonemal complex. Genes Cells 2: 487-498.

Zickler, D. and Kleckner, N. 1999. Meiotic chromosomes: Integrating structure and function. Annu. Rev. Genet. 33: 603-754. 


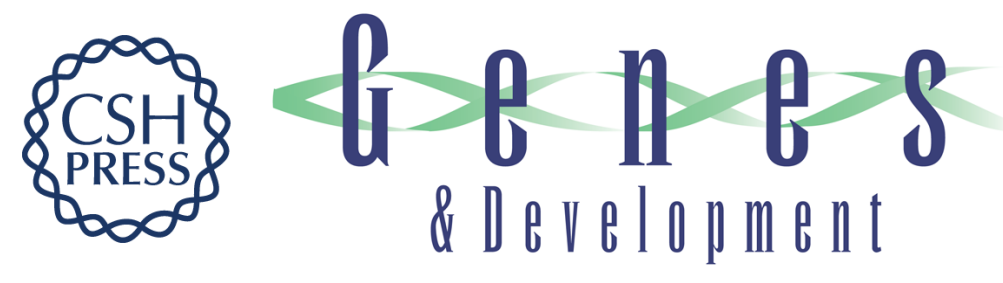

\section{Budding yeast Hed1 down-regulates the mitotic recombination machinery when meiotic recombination is impaired}

Hideo Tsubouchi and G. Shirleen Roeder

Genes Dev. 2006, 20:

Access the most recent version at doi:10.1101/gad.1422506

Supplemental http://genesdev.cshlp.org/content/suppl/2006/06/09/20.13.1766.DC1
Material

References This article cites 50 articles, 23 of which can be accessed free at:

http://genesdev.cshlp.org/content/20/13/1766.full.html\#ref-list-1

License

Email Alerting Receive free email alerts when new articles cite this article - sign up in the box at the top

Service right corner of the article or click here.

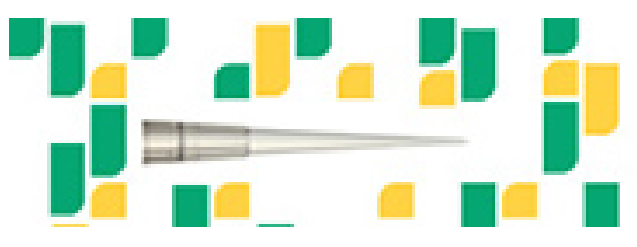

Focused on your science. 